\title{
The Role of Specialty Tubes in Preventing Ventilator-Associated Events
}

\author{
Thomas Piraino
}

\author{
Introduction \\ Ventilator-Associated Events \\ Silver-Coated Endotracheal Tubes \\ Biofilm Removal: The Mucus Shaver \\ Subglottic Secretion Drainage Tubes \\ Endotracheal Tube With Tapered Cuffs \\ Cuff Pressure Management \\ The Role of Specialty Tubes \\ Summary
}

\begin{abstract}
There have been many innovations to the standard endotracheal tube over the years, many of which were intended to reduce the incidence of ventilator-associated pneumonia (VAP). Ventilator-associated events are associated with the objective outcomes of increased duration of mechanical ventilation, length of ICU and hospital stay, and increased risk of mortality. Many specialty tubes have been associated with a reduction in the clinical diagnosis of VAP, but studies have failed to show differences in objective outcomes. This article reviews the evidence related to specialty tubes and discusses their role in improving objective outcomes associated with ventilator-associated events. Key words: ventilator-associated pneumonia; ventilator-associated events; endotracheal tubes; mechanical ventilation; patient outcomes. [Respir Care 2019;64(8):980-985. (C) 2019 Daedalus Enterprises]
\end{abstract}

\section{Introduction}

The placement of an endotracheal tube (ETT) into the airway maintains an open glottis and hinders the ability to properly clear secretions from the airway. These issues, combined with heavy sedation and immobilization, create the potential for pneumonia to occur. Pneumonia occurring after $48 \mathrm{~h}$ of invasive mechanical ventilation is re-

Mr Piraino is affiliated with St Michael's Hospital, Toronto, Ontario Canada.

Mr Piraino discloses relationships with Dräger, Philips, and Fisher \& Paykel.

Mr Piraino presented a version of this paper at the New Horizons Symposium: Ventilator-Associated Events - Surveillance and Understanding of the AARC Congress 2018, held December 4-7, 2018 in Las Vegas, Nevada. ferred to as ventilator-associated pneumonia (VAP) and has been the subject of study for well over a decade. Epidemiological studies of VAP have found associations with higher mortality, increased duration of mechanical ventilation, and increased length of hospital stay, all of which contribute to a perceived increase in the health care costs associated with length hospital stay. These associations have led clinicians to misinterpret VAP as a cause of these poor outcomes. Unfortunately, these studies often ignore the timing of the VAP event, leading to what is referred to as a time-dependent bias. ${ }^{1,2}$ In general terms, a

\footnotetext{
Correspondence: Thomas Piraino RRT FCSRT, Street Michael's Hospital, 30 Bond Street, Toronto, Ontario M5B 1W8, Canada. E-mail: thomaspiraino@gmail.com.
}

DOI: $10.4187 /$ respcare. 07048 


\section{Specialty Tubes for VAE Prevention}

Table 1. Challenges Related to Diagnostic Criteria for VAP

VAP surveillance definitions are complicated and subjective.

VAP surveillance is time-consuming and frustrating.

Surveillance is very difficult to automate.

Clinicians and infection preventionists frequently disagree about VAP diagnosis.

Clinicians and infection preventionists are often wrong relative to histology.

Clinicians and infection preventionists demonstrate high inter-observer variability.

VAP rates are lowered easily by applying surveillance criteria more strictly.

Diagnosis and treatment are susceptible to gaming.

Attributable mortality is very low.

Very few VAP prevention initiatives have decreased length of stay or lowered mortality rates.

Focusing on VAP prevention alone only addresses a small fraction of the potential harm that can befall ventilated patients.

Comparison of VAP rates between institutions is difficult or impossible.

From Reference 4.

$\mathrm{VAP}=$ ventilator-associated pneumonia

time-dependent bias treats a patient who presents with VAP during invasive mechanical ventilation as a patient who walked into the hospital with VAP, associating the entire stay and cost of care to VAP. ${ }^{1}$ The quest to reduce VAP has focused on a reduction of these associated objective outcomes, but the evidence falls short of demonstrating such benefit. Studies that report success in reducing the incidence of VAP have been unsuccessful in reducing mortality, duration of mechanical ventilation, and length of ICU and hospital stay. ${ }^{3,4}$ This calls into question the true benefits related to VAP prevention strategies that require costly interventions, due to the assumption that per-patient costs will be reduced if VAP is prevented.

The diagnostic criteria for VAP originally described by the Centers for Disease Control and Prevention have been used inconsistently across studies. ${ }^{5}$ Many of the criteria used to determine VAP are subjective or include tests known to have significant rates of false positives or false negatives. ${ }^{3}$ Commonly used criteria include worsening gas exchange, tachypnea or dyspnea, changes in sputum characteristics, rales or bronchial breath sounds, suspicion of infection, new infiltrate on chest radiograph, and pathogen growth as determine with microbiology samples. These largely subjective criteria present various challenges, including intra-observer variability (Table 1). Worsening gas exchange can have many causes, and microbiology samples have a sensitivity range of $50-70 \%$ and specificity of 40-95\% compared to tissue biopsy (the accepted standard). ${ }^{3}$ Sensitivity (the ability to identify a true positive) can be limited by a failure to sample from the affected lung segment or by diminished bacterial growth due to prior exposure to antibiotics. Specificity (the ability to identify a true negative) can be limited by contamination of the sample from the ETT or the upper airway when a patient is ventilated for longer periods of time. ${ }^{3}$

\section{Ventilator-Associated Events}

The clinical presentation of VAP shares important features with other conditions encountered in the ICU. Pulmonary edema, atelectasis, pneumonia, and ARDS all share similarities in clinical presentation, and distinguishing between them may be challenging. One important similarity between the management of these conditions is the potential need for increasing PEEP and/or $\mathrm{F}_{\mathrm{IO}_{2}}$. The increase of these settings generally indicates increased severity of illness, further distancing a patient from ventilator liberation and thus prolonging mechanical ventilation. In 2012, Klompas and colleagues ${ }^{6}$ published the use of a streamlined VAP definition, replacing the subjective pulmonary signs related to sputum and worsening gas exchange with objective thresholds of increasing PEEP and $\mathrm{F}_{\mathrm{IO}_{2}}$ after $2 \mathrm{~d}$ of clinical stability, as well as objective criteria for signs of infection (eg, blood cell counts). This streamlined approach was easier and it was reproducible, and surveillance took significantly less time to perform (3.5 min vs 39 min per patient) and had much better agreement between surveyors $($ kappa $=0.79$ vs 0.45$) .{ }^{6}$ Shortly thereafter, the Centers for Disease Control and Prevention changed their recommendations from VAP surveillance to ventilator-associated event (VAE) surveillance, which included these objective criteria. VAEs are classified when, after a period of stability or improvement, an increase in PEEP or $\mathrm{F}_{\mathrm{IO}_{2}}$ requirements by a specific amount (PEEP change of $3 \mathrm{~cm} \mathrm{H}_{2} \mathrm{O}$ or more, $\mathrm{F}_{\mathrm{IO}_{2}}$ change of 0.20 or more). If the increase is transient and settings can be reduced in $<2 \mathrm{~d}$, there is no further classification. However, if the increase is sustained (or higher) for $\geq 2 \mathrm{~d}$, it is considered a ventilator-associated condition (VAC). Further classification along the pathway depends upon other criteria regarding the likelihood of infection as shown in Figure 1. Unlike VAP, the more objective VAE criteria have consistently demonstrated associations with poor patient outcomes, including increased mortality, duration of mechanical ventilation, and length of ICU and hospital stay. ${ }^{7-9}$

One of the potential causes of VAP discussed in the literature involves the presence and function of an ETT. This has led to several innovations applied to the standard ETT. The most common innovations have focused on minimizing or preventing aspiration of oral secretions into the airways and preventing biofilm growth and transmission into the ETT. This article reviews the various ETT innovations and presents the relevant research data associated with their performance. Most of the studies to date have focused on the prevention of VAP rather than VAE, but 


\section{SPecialty Tubes for VAE PReVention}

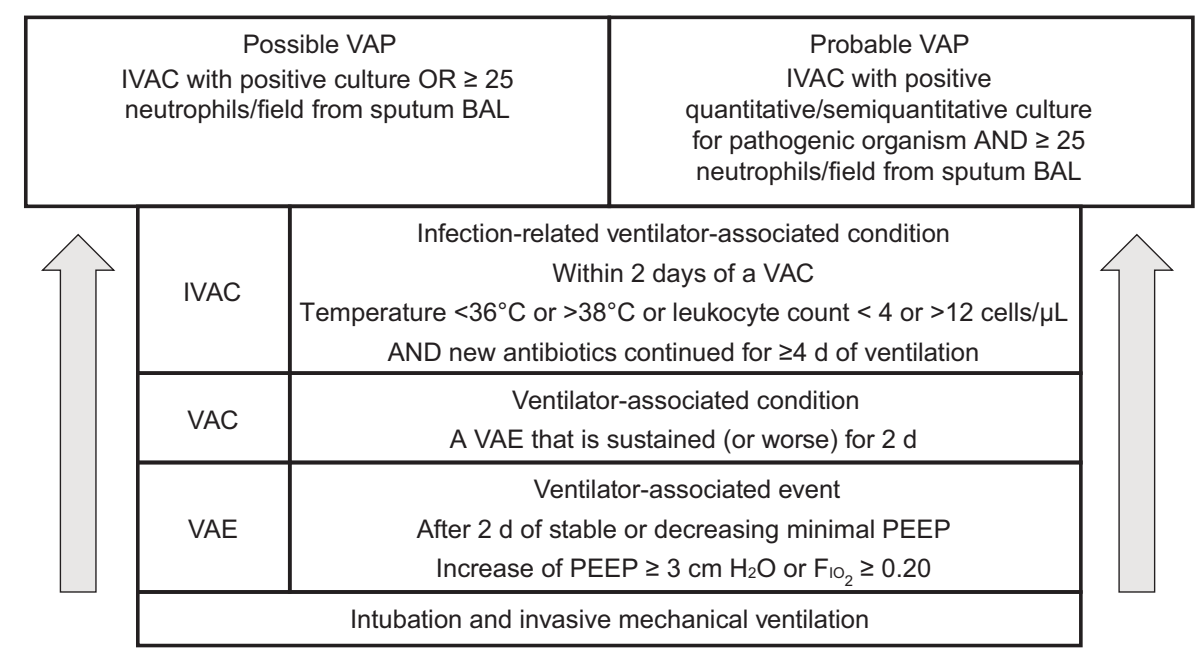

Fig. 1. Progression through ventilator-associated conditions. VAP $=$ ventilator-associated pneumonia, IVAC $=$ infection-related ventilatorassociated condition, $\mathrm{BAL}=$ bronchoalveolar lavage, $\mathrm{VAC}=$ ventilator-associated condition, $\mathrm{VAE}=$ ventilator-associated event.

the differences between VAP studies and VAE studies may provide insight into how specialty tubes may or may not contribute to the prevention of VAEs.

\section{Silver-Coated ETTs}

The ETT is a prime source of bacterial growth, and biofilm growth can begin within 3.5 days. ${ }^{10}$ One ETT innovation has been to coat the interior lining of the ETT with silver. Silver has been used topically with skin injuries to prevent infection, and in small amounts it is considered non-toxic. Silver has broad-spectrum antibacterial properties that have been reported to reduce growth and adhesion of biofilm along the interior of the ETT in animals and in a small randomized study in humans. ${ }^{11}$ This work was followed by the North American Silver-Coated Endotracheal Tube (NASCENT) Study, published in 2008. ${ }^{12}$ The NASCENT study was a prospective, multicenter, single-blind, randomized controlled study in subjects requiring mechanical ventilation for a minimum of $24 \mathrm{~h}$. A total of 1,509 subjects were included in the primary efficacy analysis, and $>1,932$ subjects were included in the safety analysis. Microbiology confirmed that VAP occurred in $4.8 \%$ of subjects in the silver-coated ETT group and in $7.5 \%$ of subjects in the uncoated group. This resulted in a $35.9 \%(95 \%$ CI 3.6-69.0) risk reduction in the incidence of VAP. However, they found no significant difference in the duration of intubation, the length of ICU or hospital stay, or mortality.

In 2010, Afessa and colleagues preformed a retrospective analysis of the NASCENT trial. ${ }^{13}$ The results of this exploratory analysis included some unexpected findings. In subjects who had VAP, mortality was lower in the silver-coated ETT group compared to controls (14\% vs $36 \%$ ). While it may appear that the use of silver-coated
ETTs reduced the burden of VAP and led to lower mortality in subjects who had VAP, the mortality rate in the silver-coated group that did not have VAP was $17 \%$ higher (14\% mortality the for silver-coated group with VAP vs $31 \%$ mortality for the silver-coated group without VAP). VAP onset $>4 \mathrm{~d}$ of mechanical ventilation was independently associated with a reduced risk of mortality (odds ratio $0.34,95 \%$ CI $0.12-0.99$ ). In subjects who did not have VAP, the mortality rate in those treated with silvercoated ETTs was also higher compared to the control group, and this was statistically significant (31\% silver-coated vs $26 \%$ control, $P=.03$ ). These finding add a level of uncertainty around the benefits of silver-coated ETTs, particularly if they reduce mortality only if you get the infection they are intended to prevent.

\section{Biofilm Removal: The Mucus Shaver}

As previously mentioned, the gradual buildup of biofilm in the ETT begins shortly after intubation. ${ }^{10}$ It has been a source of increased airway resistance, often resulting in an exchange of the ETT or insertion of a tracheostomy, and it is considered a possible cause of VAP infection among intubated patients. ${ }^{10,14}$ The removal of this biofilm was the purpose behind the development of the Mucus Shaver, which is not a type of ETT but is a catheter that has an inflatable balloon with rings around it. The catheter is inserted just beyond the distal tip of an ETT; the balloon is inflated, and the catheter is pulled through the ETT over 3-6 s, allowing the "shaving rings" to remove the accumulated biofilm from the inside of the ETT. ${ }^{15}$ To date there are limited data regarding the benefits of this device. A small, randomized controlled trial in 24 subjects demonstrated a significant reduction in biofilm with ETT cleaning performed every $6 \mathrm{~h}$ (ie, suctioning followed by clean- 


\section{Specialty Tubes for VAE Prevention}

ing with the Mucus Shaver). ${ }^{15}$ There were also significantly fewer positive microbiology cultures from the treatment group. However, the study was not powered to show a difference in the incidence of VAP or days of ventilation, which both lacked statistically significant differences, nor did they assess VAEs. Further studies are required to confirm the usefulness of this device.

\section{Subglottic Secretion Drainage Tubes}

Possibly the most studied ETT innovation related to VAP prevention is the subglottic secretion drainage tube. ${ }^{16-18}$ The trajectory of research surrounding outcomes related to the use of subglottic secretion drainage tubes is worth noting, particularly if major, systemic, hospital-wide changes were implemented after the results of the first or even second major systematic review and metaanalysis. ${ }^{16,17}$ The early systematic review and meta-analysis by Dezfulian and colleagues ${ }^{16}$ in 2005 included 5 RCTs involving 896 subjects. Their analysis reported a reduction of approximately $50 \%$ in the incidence of VAP, a 2-d reduction in mechanical ventilation, and a 3 -d reduction in ICU stay in favor of subglottic secretion drainage tubes. The investigators reported no difference in mortality and a high heterogeneity between studies. In 2011, Muscedere and colleagues ${ }^{17}$ published a systematic review and metaanalysis of 13 studies involving 2,442 subjects. They also reported a reduction of approximately 50\% in VAP incidence. They noted that ICU length of stay and duration of mechanical ventilation were lower with the use of subglottic secretion drainage tubes, although there was high heterogeneity between studies (eg, $\mathrm{I}^{2}=77 \%$ for ICU length of stay, $\mathrm{I}^{2}=86 \%$ for duration of mechanical ventilation). High heterogeneity between studies makes it extremely difficult to answer questions with certainty. Because of this uncertainty, the investigators concluded that use of subglottic secretion drainage tubes reduces VAP and may improve other clinical outcomes.

One of the issues with these earlier systematic reviews and meta-analyses was that they included results from a study published in 1995 by Valles and colleagues. ${ }^{18}$ In this study, the results showed no difference in length of ICU stay or duration of mechanical ventilation, but meta-analyses had misinterpreted the results, abstracting median values as mean values, which resulted in significant differences between groups. After this discovery, Caroff and colleagues ${ }^{19}$ performed an updated systematic review and meta-analysis in 2016 to assess objective outcomes associated with the use of subglottic secretion drainage tubes, including VAEs in the analysis. They included the study by Valles et $\mathrm{al}^{18}$ for the analysis of VAP incidence but excluded it from the analysis of length of ICU stay and duration of mechanical ventilation because the author no longer had the data to allow proper analysis of these out- comes. The analysis of 17 RCTs involving 3,369 subjects again reported a significant reduction in VAP with use of subglottic secretion drainage tubes, no mortality benefit, and no difference in length of ICU or hospital stay, and no difference in duration of mechanical ventilation. The only study assessing VAP and VAE found a significant reduction in VAP, but no difference in VAEs along with no difference in any other objective clinical outcome. ${ }^{20}$ As already mentioned, VAEs have consistently been associated with clinical outcomes such as ICU stay, duration of mechanical ventilation, and increased mortality. ${ }^{7-9}$

These results highlight the issues with VAP as a quality indicator. If VAP was the cause of poor outcomes leading to increased hospital burden and cost, then reducing VAP should demonstrate a reduction in outcomes associated with this burden. Having a clinical diagnosis of VAP does not change cost-related outcomes.

\section{ETTs With Tapered Cuffs}

One of the other innovations made to ETTs is the shape of the balloon cuff. When traditional cuffed ETTs are inflated, longitudinal folds may be present in areas where the cuff could not fully inflate due to the size of the trachea. The channels along the folds are potential areas for microaspiration of oral secretions. A tapered shape, theoretically, would at some point be the same diameter as the airway and therefore have no longitudinal folds to allow oral secretions to pass. In vitro animal and bench studies have reported mixed results. ${ }^{21-23}$ However, a recent systematic review and meta-analysis of studies using these tubes failed to indicate any difference in VAP between tapered and standard ETT cuffs. Furthermore, the addition of a subglottic secretion drainage tube to a tapered ETT cuff, assessed in a prospective randomized controlled trial of 276 subjects, had results similar to previous studies of subglottic secretion drainage tubes, namely a significant reduction in VAP diagnosis (specifically late-onset VAP) but no difference in other objective outcomes. ${ }^{24}$

\section{Cuff Pressure Management}

Another possible solution for preventing VAP is the control and close monitoring of ETT cuff pressures. A recent study by Letvin and colleagues ${ }^{25}$ assessed the impact of cuff pressure monitoring frequency on the occurrence of VAP and VAEs. They compared cuff pressure assessment immediately after intubation for all subjects, then assigned frequent (every $8 \mathrm{~h}$ and when clinically indicated) or only when clinically indicated cuff pressure monitoring based on which ICU bed they were admitted to. The goal was to maintain a cuff pressure $24-30 \mathrm{~cm}$ $\mathrm{H}_{2} \mathrm{O}$ when measured. A total of 305 subjects were enrolled in the study and there was no difference between groups 


\section{Specialty Tubes for VAE Prevention}

regarding VAP diagnosis or VAEs. In 2014, Lorente and colleagues ${ }^{26}$ investigated the impact of continuous versus intermittent cuff pressure maintenance on the occurrence of VAP. In this study ( $N=284$ subjects), all subjects received ETTs with subglottic secretion drainage and were assigned continuous or intermittent (every $8 \mathrm{~h}$ ) maintenance of ETT cuff pressure at $25 \mathrm{~cm} \mathrm{H}_{2} \mathrm{O}$. The continuous monitoring of cuff pressure in this study resulted in significantly less VAP $(P=.02)$ but no difference in the other objective outcomes measured, including duration of mechanical ventilation and ICU mortality.

\section{The Role of Specialty Tubes}

A critical appraisal of the available evidence regarding specialty tubes reveals significant concerns about the value of VAP as a quality indicator or prevention target. Subjective criteria combined with tests with wide ranges of false positives and false negatives is the main reason VAEs were created. If the purpose of these specialty tubes is to reduce the incidence of infection diagnosed by these criteria, they succeed. But what are specialty tubes reducing if the burden of caring for these patients (objective outcomes) is not altered? If a clinician were to weigh the cost associated with these specialty tubes or monitoring systems against the benefit (ie, value), these tubes do not hold clinically relevant value. Without affecting objective clinical outcomes such as duration of mechanical ventilation, length of ICU and hospital stay, or mortality, where is the cost savings in the implementation of specialty tubes as part of a VAP prevention bundle?

\section{Summary}

There are limited studies that specifically look at VAE and specialty ETTs. However, when VAP and VAEs have been compared in studies of specialty ETTs, reduction in VAP does not result in a difference in VAEs or in objective clinical outcomes such as mortality, duration of mechanical ventilation, or length of ICU or hospital stay. This means that the lack of difference in objective outcomes between groups is reflected in the VAE results, but not in the incidence of VAP. The reduction of objective outcomes would result in a decreased burden of care and cost to hospitals, and the current evidence suggests that specialty ETTs have failed to demonstrate this value.

\section{REFERENCES}

1. Beyersmann J, Gastmeier P, Wolkewitz M, Schumacher M. An easy mathematical proof showed that time-dependent bias inevitably leads to biased effect estimation. J Clin Epidemiol 2008;61(12):1216-1221.

2. van Walraven C, Davis D, Forster AJ, Wells GA. Time-dependent bias was common in survival analyses published in leading clinical journals. J Clin Epidemiol 2004;57(7):672-682.
3. Klompas M. The paradox of ventilator-associated pneumonia prevention measures. Crit Care 2009;13(5):315.

4. Klompas M, Berra L. Should ventilator-associated events become a quality indicator for ICUs? Respir Care 2016;61(6):723-736.

5. Horan TC, Andrus M, Dudeck MA. CDC/NHSN surveillance definition of health care-associated infection and criteria for specific types of infections in the acute care setting. Am J Infect Control 2008;36(5):309-332.

6. Klompas M, Kleinman K, Khan Y, Evans RS, Lloyd JF, Stevenson $\mathrm{K}$, et al. Rapid and reproducible surveillance for ventilator-associated pneumonia. Clin Infect Dis 2012;54(3):370-377.

7. Hayashi Y, Morisawa K, Klompas M, Jones M, Bandeshe H, Boots $\mathrm{R}$, et al. Toward improved surveillance: the impact of ventilatorassociated complications on length of stay and antibiotic use in patients in intensive care units. Clin Infect Dis 2013;56(4):471-477.

8. Klompas M, Kleinman K, Murphy MV. Descriptive epidemiology and attributable morbidity of ventilator-associated events. Infect Control Hosp Epidemiol 2014;35(5):502-510.

9. Muscedere J, Sinuff T, Heyland DK, Dodek PM, Keenan SP, Wood $\mathrm{G}$, et al. The clinical impact and preventability of ventilator-associated conditions in critically ill patients who are mechanically ventilated. Chest 2013;144(5):1453-1460.

10. Coppadoro A, Thomas JG, Berra L. Endotracheal tube biofilm and ventilator-associated pneumonia. In: Vincent J-L, editor. Annual update in intensive care and emergency medicine 2013. Berlin, Heidelberg: Springer Berlin Heidelberg; 2013:579-587.

11. Rello J, Kollef M, Diaz E, Sandiumenge A, del Castillo Y, Corbella X, et al. Reduced burden of bacterial airway colonization with a novel silver-coated endotracheal tube in a randomized multiple-center feasibility study. Crit Care Med 2006;34(11):2766-2772.

12. Kollef MH, Afessa B, Anzueto A, Veremakis C, Kerr KM, Margolis $\mathrm{BD}$, et al. Silver-coated endotracheal tubes and incidence of ventilator-associated pneumonia: the NASCENT randomized trial. JAMA 2008;300(7):805-813.

13. Afessa B, Shorr AF, Anzueto AR, Craven DE, Schinner R, Kollef $\mathrm{MH}$. Association between a silver-coated endotracheal tube and reduced mortality in patients with ventilator-associated pneumonia. Chest 2010;137(5):1015-1021.

14. Rumbak MJ, Walsh FW, Anderson WM, Rolfe MW, Solomon DA. Significant tracheal obstruction causing failure to wean in patients requiring prolonged mechanical ventilation: a forgotten complication of long-term mechanical ventilation. Chest 1999;115(4):1092-1095.

15. Berra L, Coppadoro A, Bittner EA, Kolobow T, Laquerriere P, Pohlmann JR, et al. A clinical assessment of the Mucus Shaver: a device to keep the endotracheal tube free from secretions. Crit Care Med 2012;40(1):119-124.

16. Dezfulian C, Shojania K, Collard HR, Kim HM, Matthay MA, Saint S. Subglottic secretion drainage for preventing ventilator-associated pneumonia: a meta-analysis. Am J Med 2005;118(1):11-18.

17. Muscedere J, Rewa O, McKechnie K, Jiang X, Laporta D, Heyland DK. Subglottic secretion drainage for the prevention of ventilatorassociated pneumonia: a systematic review and meta-analysis. Crit Care Med 2011;39(8):1985-1991.

18. Valles J, Artigas A, Rello J, Bonsoms N, Fontanals D, Blanch L, et al. Continuous aspiration of subglottic secretions in preventing ventilator-associated pneumonia. Ann Intern Med 1995;122(3):179-186.

19. Caroff DA, Li L, Muscedere J, Klompas M. Subglottic secretion drainage and objective outcomes: a systematic review and metaanalysis. Crit Care Med 2016;44(4):830-840.

20. Damas P, Frippiat F, Ancion A, Canivet JL, Lambermont B, Layios $\mathrm{N}$, et al. Prevention of ventilator-associated pneumonia and ventilator-associated conditions: a randomized controlled trial with subglottic secretion suctioning. Crit Care Med 2015;43(1):22-30. 


\section{Specialty Tubes for VAE Prevention}

21. Zanella A, Scaravilli V, Isgro S, Milan M, Cressoni M, Patroniti N, et al. Fluid leakage across tracheal tube cuff, effect of different cuff material, shape, and positive expiratory pressure: a bench-top study. Intensive Care Med 2011;37(2):343-347.

22. Li Bassi G, Luque N, Marti JD, Aguilera Xiol E, Di Pasquale M, Giunta V, et al. Endotracheal tubes for critically ill patients: an in vivo analysis of associated tracheal injury, mucociliary clearance, and sealing efficacy. Chest 2015;147(5):1327-1335.

23. Chenelle CT, Itagaki T, Fisher DF, Berra L, Kacmarek RM. Performance of the PneuX system: a bench study comparison with 4 other endotracheal tube cuffs. Respir Care 2017;62(1):102-112.
24. Mahmoodpoor A, Hamishehkar H, Hamidi M, Shadvar K, Sanaie S, Golzari SE, et al. A prospective randomized trial of tapered-cuff endotracheal tubes with intermittent subglottic suctioning in preventing ventilator-associated pneumonia in critically ill patients. J Crit Care 2017;38:152-156.

25. Letvin A, Kremer P, Silver PC, Samih N, Reed-Watts P, Kollef MH. Frequent versus infrequent monitoring of endotracheal tube cuff pressures. Respir Care 2018;63(5):495-501.

26. Lorente L, Lecuona M, Jimenez A, Lorenzo L, Roca I, Cabrera J, et al. Continuous endotracheal tube cuff pressure control system protects against ventilator-associated pneumonia. Crit Care 2014;18(2):R77. 